\title{
A Planar Filter-Lens Array For Millimeter-Wave Applications
}

\author{
Abbas Abbaspour-Tamijani , Kamal Sarabandi, and Gabriel M. Rebeiz
}

The University of Michigan, Radiation Laboratory

Ann Arbor, MI 48109-2122 USA

FAX: +1-734-647-2106 email: abbasa@umich.edu

\section{INTRODUCTION}

Planar lens arrays have been addressed by a number of researchers in the existing literature [1][2] [3][4]. In these works, focusing is obtained by using arrays of receive and transmit antennas which are connected by transmission-line sections of appropriate lengths. Presence of these line sections has adverse effects on the frequency-response and complicates the layout design for large arrays. An alternative approach can be developed based on the non-uniform arrays of antenna-filter-antenna (AFA) elements. These elements have been previously demonstrated and used in periodic (uniform) arrays for designing frequency-selective surfaces (FSS) [5]. Using different types of bandpass AFA elements with different values of phase-delay in an array results in a lens-like FSS structure, which can be referred to as a filter-lens array (FLA). Scaled versions of a 3-pole bandpass AFA can achieve in-band phase transitions between 0 and 180 degrees, which is enough for small lenses. An arbitrary phase-transition of 0 to 360 degrees can be obtained by combining the scaled versions of two different types of AFA elements.

\section{FREQUENCY RESPONSE OF BANDPASS AFA ELEMENTS}

A 3-pole bandpass AFA element is composed of two back-to-back patch antennas and a co-planarwaveguide (CPW) resonator which lies in the common ground plane. Each patch antenna can be considered as the combination of a lossless resonator and a resistive load, representing the radiation and internal losses [6]. If the radiation part is considered as the external loading and the internal losses are attributed to the resonator, the AFA structure can be viewed as a bandpass filter between two radiation ports (Fig. 1).

The above-described filter model can be used to design different AFA elements. Two types of AFA elements are considered for designing a general FLA, referred to as Type-I and Type-II elements. Layouts and circuit models of these two types of elements are shown in Fig. 2 . In these AFA structures, the antenna elements are hexagonal patches which are made on $500 \mu \mathrm{m}$ thick glass substrates with $\varepsilon_{r}=4.45$ and $\tan \delta \approx 0.06$. In Type-I AFA, the middle resonator is a quarter-wave CPW resonator, which is fabricated in the common ground plane. The quarter-wave resonator couples to the patch antennas at its open end. In Type-II element the center resonator is a deformed half-wave resonator which couples to the top and bottom patches with $180^{\circ}$ phase difference at its flared ends. The described AFA structures are single-polarized elements and present the required frequency response only for the incident waves polarized in parallel with the CPW resonators. The frequency response of the AFA elements can be controlled by appropriate choice of the layout dimensions, which in turn are obtained from the circuit models and filter design methods described in [5]. Fig. 3 shows the simulated amplitude and phase of the transmission frequency-response for the AFA elements designed for $8 \%$ bandwidth at $35 \mathrm{GHz}$. The in-band phase-delay between the first and third peaks of the amplitude response $\left(-\angle S_{21}\right)$ varies from $81^{\circ}$ to $268^{\circ}$ in Type-I element, and from $-67^{\circ}$ to $117^{\circ}$ in Type-II element. 


\section{FILTER-LENS ARRAY}

In a Filter-Lens Array, the wave-front transformation is performed by the comprising AFA elements, which enforce the desired phase-shift between the input and output of each array cell. To achieve different values of phase-shift, AFA elements are de-tuned in the vicinity of the operating frequency. The de-tuned elements are obtained from scaling the transversal dimensions in the a tuned design (with bandwidth centered at the operating frequency). The maximum range of phase shift that can be obtained in this way is equal to the in-band variations of the phase-delay, which is nearly $180^{\circ}$ in case of a 3-pole AFA. This range can be increased by using two types of elements in the array. For the combination of the Type-I and Type-II elements, for example, this range is equal to $-268^{\circ}$ to $67^{\circ}$, which is practically sufficient for realizing any desired wave-transformation.

To demonstrate this concept, a 137-element FLA was designed based on Type-I and Type-II elements for operation at $35 \mathrm{GHz}$ and with a focal distance of $f=10 \mathrm{~cm}$ (Fig 4a), and was fabricated on 3-inch glass wafers. For each cell $m$, the element type $I_{m}$ and scaling factor $\alpha_{m}$ are chosen to provide the required phase-shift $\Phi_{m}$ (Fig. 4). For the cells where both types of elements can be used, the element types are chosen based on the minimum values of insertion loss and required scaling $\left(\left|\alpha_{m}-1\right|\right)$.

A simple analysis can be performed to asses the performance of the designed FLA. The proposed analysis is based on the ray-tracing approach, and makes three assumptions: 1) the antenna elements in different cells do not interact, 2) the feed and AFA elements are polarization-matched, and 3) the frequency responses of the AFA elements remain unchanged for oblique angles of incidence. While the first two assumptions are generally true for most FLA designs, the last assumption is valid only for FLA's with the small subtended angles $(f / D \geq 1)$. Assuming that the FLA is fed by an antenna feed of known pattern at its focal point, the analysis starts with calculating the excitation coefficients of the AFA elements at the input aperture. Output array coefficients are then obtained by applying the transfer functions of the individual elements. The FLA power transfer ratio, gain, and radiation pattern can be calculated from these array coefficients.

The calculated and measured gain patterns of the 3-inch FLA are presented in Fig. 5, for E-and H-planes ( $\varphi=0$ and $90^{\circ}$ respectively). Although an open-ended waveguide feed was used for the FLA measurements, the presented results are re-normalized for an ideal feed by de-embedding the spill-over loss and feed antenna efficiency from the measured gain. The measured values of the maximum gain and 3- $\mathrm{dB}$ beam-width are $25.6 \mathrm{dBi}$ and $6.4^{\circ}$ (in both planes), vs. the simulated values of $24.8 \mathrm{dBi}$ and $7.0^{\circ}$, respectively. The measured sidelobe level is $-14 \mathrm{~dB}$ in the E-plane and $-17 \mathrm{~dB}$ in the H-plane.

Figure 6 presents the calculated and measured maximum gain of the 3-inch FLA as a function of frequency. According to these results, the designed FLA presents a gain bandwidth of $8.2 \%$ at $35.3 \mathrm{GHz}$. Considering that a 3 inch circular uniform aperture would have a theoretical gain of $29.1 \mathrm{dBi}$ at $35 \mathrm{GHz}$, this FLA can be considered as the combination of an ideal lens and a bandpass filter with the mid-band insertion loss of $3.5 \mathrm{~dB}$. Table 1 summarizes the important performance data for this design.

Table 1: Measured Performance Data for The 137-Element FLA.
\begin{tabular}{|l|c|}
\hline Center Frequency (GHz) & 35.25 \\
\hline \hline Mid-Band Maximum Gain (dBi) & 25.6 \\
HPBW (deg.) & 6.4 \\
SLL E-Plane (dB) & -14 \\
SLL H-Plane (dB) & -17 \\
\hline \hline Equivalent Mid-band Directivity (dBi) & 29.1 \\
Equivalent Mid-band Insertion-Loss (dB) & 3.5 \\
3-dB Gain Bandwidth & $8.2 \%$ \\
20-dB Rejection-Band Edges (GHz) & $32,38.8$ \\
\hline Total Power Efficiency & $45 \%$ \\
\hline
\end{tabular}




\section{CONCLUSION}

The novel concept of Filter-Lens Array was introduced in this paper. Measured results for a $\mathrm{Ka}$ band design show that a typical FLA can meet system requirements for a wide range of applications. FLA's present a compact and simple means of focusing and filtering at millimeter-wave frequencies, and can replace the standard combinations of dielectric lenses and bandpass filters. They are expected to find application in power combining, medium-angle beam-steering, and millimeter-wave imaging systems. Scenarios can be envisioned for the integration of active or MEMS devices in FLA structures, for the applications where additional gain or adaptive beam-forming are required.

\section{REFERENCES}

[1] D. T. McGrath, "Planar three-dimentional constrained lens," IEEE Trans. Antennas and Propagation, vol. 34, pp. 46-50, Jan. 1986.

[2] D. M. Pozar, "Flat lens antenna concept using aperture coupled microstrip patches," Electronic Letters, vol. 32, pp. 2109-2111, Nov. 1996.

[3] D. Popovic and Z. Popovic, "Multibeam antennas with polarization and angle diversity," IEEE Trans. Antennas and Propagation, vol. 50, pp. 651-657, May 2002.

[4] S. Romisch, D. Popovic, N. Shino, R. Lee, and Z. Popovic, "Multi-beam discrete lens arrays with amplitude-controlled steering," in 2003 IEEE MTT-S Int. Microwave Symp. Dig., Philadelphia, PA, June 2003, pp. 1669-1672.

[5] A. Abbaspour-Tamijani, B. Schoenlinner, and G. M. Rebeiz, "A new class of bandpass frequency selective structures," in 2003 AP-S Int. Symp. Dig., Columbus, $\mathrm{OH}$, June 2003, pp. $817-820$.

[6] W. L. Stutzman, Antenna Theory and Design, 2nd Edition, John Wiley and Sons, Hoboken, NJ, 1997.

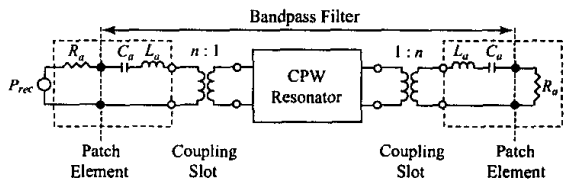

Figure 1: Antenna-Filter-Antenna element as a bandpass filter with radiation ports.

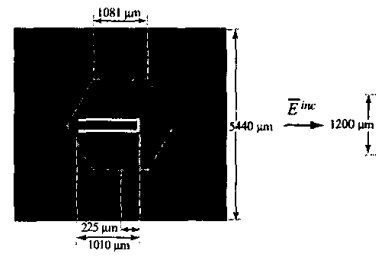

(a)

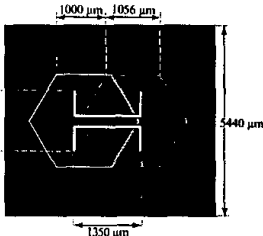

(b)

Figure 2: Layouts of two $35 \mathrm{GHz}$ AFA elements: a) Type-I, b) Type-II. 

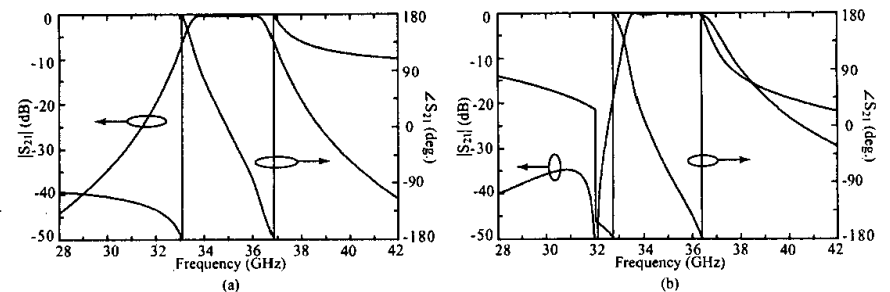

Figure 3: Simulated frequency response based on the circuit models of [5]: a) Type-I and b) Type-II elements.

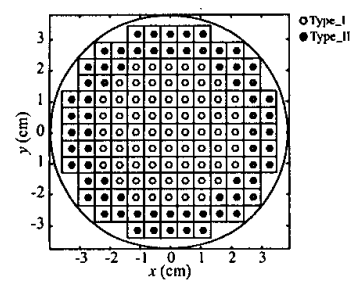

(a)

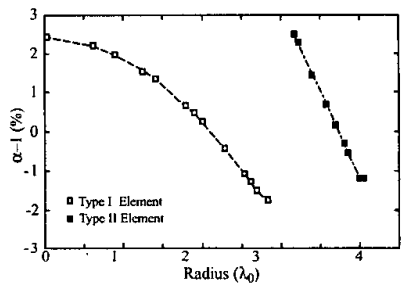

(b)

Figure 4: A 3 inch FLA using Type-I and Type-Il elements: a) Type of the array elements, b) scaling factors vs. element distance from the center of FLA.
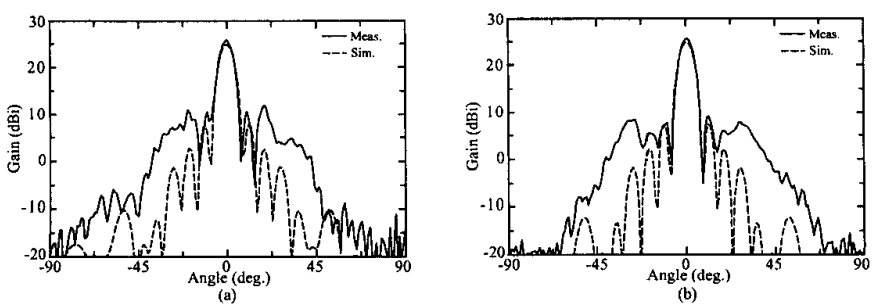

Figure 5: Measured and simulated patterns of the 137-element FLA at $35 \mathrm{GHz}$ : a) E-plane, and b) H-planes patterns.

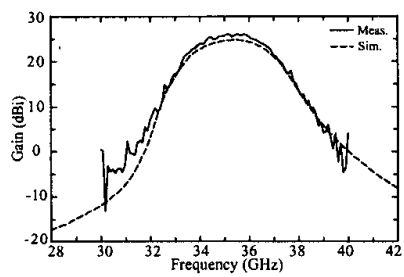

Figure 6: Measured and simulated maximum gain of the FLA vs. frequency 